\title{
Características nutricionais de doces em pasta de Araticum (Annona crassiflora Mart.)
}

\author{
Nutritional characteristics of Araticum (Annona crassiflora Mart.) preserves
}

\author{
B. B. Dias; M. O. S. Oliveira*; R. A. Morais; B. C. B de Freitas; G. A. S. Martins \\ Laboratório de Cinética e Modelagem de Processos, Universidade Federal do Tocantins, 77001-090, \\ Palmas-Tocantins, Brasil \\ *mariaoliviaeng@gmail.com \\ (Trabalho avaliado e selecionado pela Comissão do III CTOCTA)
}

\begin{abstract}
Fruto nativo do Cerrado, o Araticum (Annona crassiflora Mart.) é muito consumido in natura. Devido a sua sazonalidade a aplicação de tecnologias de processamento são alternativas que garantem o aumento de sua vida útil, e consequentemente agrega valor econômico, entre elas, uma das formas mais aceitas pelos consumidores, é a transformação dos frutos em doces. Diante disso, o conhecimento da composição nutricional, bem como o desenvolvimento da tabela de composição nutricional de acordo com a legislação é de grande importância. Portanto, o presente trabalho tem como objetivo aproveitar a polpa do Araticum na forma de doce em pasta com substituições da pectina usada comercialmente por albedo de maracujá, e ainda avaliar a qualidade nutricional de formulações, com foco na inserção do fruto no mercado consumidor. Para o processamento, foi utilizado um delineamento experimental $2^{3} \mathrm{com} 11$ ensaios e 3 variáveis independentes (ácido cítrico, polpa/açúcar e albedo de maracujá). Foi procedida a informação nutricional das formulações de acordo com as legislações vigentes e dispostas em tabelas de composição nutricional. As formulações apresentaram baixo valor calórico, valores estes, menores do que aqueles encontrados para doces comerciais, o que amplia as possibilidades de consumo do produto, como na alimentação escolar.
\end{abstract}

Palavras-chave: Araticum, composição nutricional, rotulagem.

A native fruit of the Cerrado, Araticum (Annona crassiflora Mart.) is widely consumed fresh. Due to their seasonality, the application of processing technologies are alternatives that ensure an increase in their shelf life, and consequently add economic value, among them, one of the most accepted ways by consumers is the transformation of fruits into sweets. Therefore, the knowledge of the nutritional composition, as well as the development of the nutritional composition table according to the legislation, is of great importance. Therefore, the present work has as objective the Araticum pulp in the form of candy in mass with substitutions of the pectin used commercially by passion fruit albedo, and also to evaluate the nutritional quality of formulations, focusing on the insertion of the fruit in the consumer market. For processing, a $2^{3}$ experimental design with 11 trials and 3 independent variables (citric acid, pulp/sugar and passion fruit albedo) was used. Nutritional information of the formulations was carried out in accordance with current legislation and arranged in nutritional composition tables. The low-calorie formulations, values, are lower than those found for commercial sweets, which expands the possibilities of consumption of the product, such as in school meals.

Keywords: Araticum, nutritional composition, labeling.

\section{INTRODUÇÃO}

As espécies frutíferas nativas representam uma grande riqueza dentro da biodiversidade brasileira, onde uso sustentável de seus frutos pode trazer benefícios para a melhoria da nutrição e consequentemente da saúde. O Cerrado, bioma brasileiro detêm um valioso patrimônio de recursos naturais renováveis com grande potencial para ser explorado comercialmente, pois apresenta inúmeras espécies frutíferas exóticas que apresentam características sensoriais únicas e intensas [1].

Nesse sentido, umas das fontes mais importantes de nutrientes na alimentação são os frutos, estes por sua vez se deterioram rapidamente, devido as suas atividades metabólicas elevadas [2, 3]. Muitas espécies são sazonais e não apresentam disponibilidade durante o ano inteiro, com isso, torna-se necessário que se apliquem tecnologias para que aumente a vida útil do fruto [2]. Uma das maneiras mais usuais e bem aceitas pelos consumidores para conservar as propriedades de 
frutos é o processamento sob a forma de doces [4]. A adição de açúcar e ácido cítrico é um dos métodos de conservação de frutas pelo qual a preservação ocorre pela redução da atividade de água, que evita a proliferação e o desenvolvimento de microrganismos [5].

O desenvolvimento de produtos a partir de frutos nativos eleva a importância cultural e nutricional, e resultam na geração de renda a inúmeras famílias. Através de produtos como doces o incentivo ao consumo dos frutos nativos é estimulado, pois com uma maior vida útil a comercialização em maiores períodos de tempo e a disseminação em diferentes regiões geográficas se torna viável. Diante do exposto, a necessidade do conhecimento da composição nutricional, bem como o desenvolvimento da tabela de composição nutricional de acordo com a legislação, é de grande importância para comercializar produtos dos frutos.

A rotulagem nutricional se caracteriza como a descrição contida na embalagem de um alimento com a intenção de informar o consumidor sobre as propriedades nutricionais ou composição daquele determinado alimento. Esta, deve compreender a declaração de valor energético e os principais nutrientes, e garantir que estas informações sejam entendidas por todos [6, 7]. As informações provenientes da rotulagem contemplam um direito assegurado pelo Código de Defesa do Consumidor, determinando que as informações contidas nos produtos devem ser claras, contendo a especificação correta de quantidade, composição e qualidade, e ainda alertar sobre os possíveis riscos que possam ocasionar ao consumidor [8, 9]. Diante disso, a informação nutricional, contida no rótulo do alimento é uma ferramenta útil e essencial para o desenvolvimento da educação nutricional, devido sua importância e necessidade para uma alimentação correta [7].

Nesse contexto apresenta-se o Araticum (Annona crassiflora Mart.) é um fruto nativo do bioma Cerrado, muito consumido in natura, mas que pode ser processado sob a forma de geleias, sucos, bolos, sorvetes e doces. Com isso, o presente trabalho tem como objetivo aproveitar a polpa do Araticum na forma de doce em pasta com substituições da pectina usada comercialmente por albedo de maracujá, e ainda avaliar a qualidade nutricional de formulações, com foco na inserção do fruto no mercado consumidor.

\section{MATERIAL E MÉTODOS}

\subsection{Matéria-Prima}

As polpas integrais do Araticum foram disponibilizadas pela cooperativa Grande Sertão, (Montes Claros, MG - 16²4'06.6"S, 4352'05.7"W) para o Laboratório de Cinética e Modelagem de Processos na Universidade Federal do Tocantins (UFT, Palmas, TO) através de transporte aéreo, em embalagens térmicas para evitar o descongelamento. As polpas recebidas foram armazenadas sob congelamento até o processamento.

\subsection{Processamento Dos Doces}

Para o processamento foram seguidas as etapas: recepção das polpas, pesagem dos ingredientes de cada formulação, os ingredientes foram levados a cocção, e somente próximo do ponto final o ácido foi então adicionado. O processo de cocção foi cessado quando o doce atingiu $78^{\circ}$ Brix. Em seguida, foi realizado o enchimento à quente e fechamento da embalagem. Utilizouse o albedo de maracujá como fonte de pectina [10].

\subsection{Planejamento Experimental}

Para as elaborar as formulações foi utilizado um delineamento fatorial completo $2^{3}$ [11] para gerar as proporções entre as variáveis dos ingredientes (Tabela 1). Os limites de concentração de ácido cítrico, proporção de polpa/açúcar e albedo (pectina) foram inseridos conforme é estabelecido na literatura e legislações pertinentes. 
Tabela 1: Delineamento experimental (23) para elaboração das formulações de doce de Araticum. $X 1=$ concentração de ácido cítrico (\%); X2 = razão polpa/açúcar $(\mathrm{m} / \mathrm{m})$ e X3= Concentração de albedo $(\%)$.

\begin{tabular}{ccccccc}
\hline \multirow{2}{*}{ Ensaios } & \multicolumn{3}{c}{ Variáveis codificadas } & \multicolumn{3}{c}{ Variáveis reais } \\
\cline { 2 - 7 } & $\mathbf{X 1}$ & $\mathbf{X 2}$ & $\mathbf{X 3}$ & $\mathbf{X 1}(\mathbf{\%})$ & $\mathbf{X 2}(\mathbf{m} / \mathbf{m})$ & $\mathbf{X 3}(\mathbf{\%})$ \\
\hline $\mathbf{1}$ & +1 & +1 & +1 & 1 & $60 / 40$ & 3 \\
$\mathbf{2}$ & -1 & -1 & +1 & 0 & $40 / 60$ & 3 \\
$\mathbf{3}$ & +1 & -1 & +1 & 1 & $40 / 60$ & 3 \\
$\mathbf{4}$ & -1 & +1 & +1 & 0 & $60 / 40$ & 3 \\
$\mathbf{5}$ & +1 & +1 & -1 & 1 & $60 / 40$ & 0 \\
$\mathbf{6}$ & -1 & +1 & -1 & 0 & $60 / 40$ & 0 \\
$\mathbf{7}$ & +1 & -1 & -1 & 1 & $40 / 60$ & 0 \\
$\mathbf{8}$ & -1 & -1 & -1 & 0 & $40 / 60$ & 0 \\
$\mathbf{9}$ & 0 & 0 & 0 & 0,5 & $50 / 50$ & 1,5 \\
$\mathbf{1 0}$ & 0 & 0 & 0 & 0,5 & $50 / 50$ & 1,5 \\
$\mathbf{1 1}$ & 0 & 0 & 0 & 0,5 & $50 / 50$ & 1,5 \\
\hline
\end{tabular}

\subsection{Rotulagem Nutricional}

As tabelas de informação nutricional dos doces foram elaboradas através de cálculos na ferramenta Excel, com a consulta ao Manual de Orientação às Indústrias de Alimentos [6], atendendo as legislações vigentes. São elas, a $\mathrm{RDC} \mathrm{n}^{\circ} 359$ [12] que trata do Regulamento Técnico de Porções de Alimentos Embalados Para Fins de Rotulagem Nutricional e a RDC no 360 [13] que aborda o Regulamento Técnico Sobre Rotulagem Nutricional de Alimentos Embalados, considerando as normas aprovadas para o âmbito do MERCOSUL, incorporadas na portaria $\mathrm{n}^{\circ}$ 33 [14] referente a Ingestão Diária Recomendada (IDR).

\section{RESULTADOS E DISCUSSÃO}

As informações nutricionais das formulações estão descritas nos Quadros 1 e 2. As formulações apresentaram valor entre 60 e $82 \mathrm{kcal} /$ porção, valores abaixo de doces já comercializados $[15,16]$. O Programa Nacional de Alimentação Escolar (PNAE) com o objetivo de limitar a inserção de alimentos processados de baixo valor nutricional em cardápios escolares, estabeleceu na Resolução CD/FNDE n ${ }^{0} 26$ de 17 de junho de 2013 [17] que a oferta de doces e/ou preparações doces a partir da vigência da normativa ficaria limitada a duas porções por semana, com o valor calórico equivalente a $110 \mathrm{kcal} /$ porção. Logo, as formulações desenvolvidas para os doces de Araticum podem ser consideradas alternativas viáveis para inserção na alimentação de adultos e crianças, inclusive em cardápios escolares

No entanto, como é possível observar nos quadros, os doces de Araticum não apresentaram baixo valor nutricional. Os doces apresentaram baixo teor de gordura, presença de fibras alimentares, cálcio e sódio, além de apresentarem a capacidade de serem apontados como fonte de vitamina $\mathrm{C}$ e $\mathrm{A}$. A formulação 8 apresentou a maior concentração de carboidratos e consequentemente um maior valor calórico, apresentando um maior valor energético. A formulação que demonstrou melhores valores na maioria dos parâmetros foi a Formulação 1, mas por outro lado esta mesma formulação apresentou maiores valores de sódio e gorduras.

Entre os fatos que justificam a elaboração de doces a partir da polpa do Araticum estão a presença de fibras alimentares e vitamina A. A presença das fibras alimentares nos doces desenvolvidos (Quadros 1 e 2) está relacionada a presença de oligossacarídeos na matéria-prima previamente descritos por Arruda et al. (2018) [18]. Os autores descobriram em seu estudo com a polpa do fruto Araticum, seis tipos de oligossacarídeos, os quais são considerados fibras dietéticas que exercem função prebiótica, estimulando o crescimento da microflora intestinal benéfica, tais resultados demonstram que o fruto é uma fonte potencial de produtos funcionais. Schiassi et al. (2018) [19] identificaram na polpa do fruto Araticum que as fibras alimentares 
totais compreendem $21,62 \%$, valor superior do com as frutas mais adquiridas pela população brasileira (banana prata, laranja pêra, maçã fuji, mamão e melancia).

Cardoso et al. (2013) [20] e Schiassi et al. (2018) [19] observaram que a polpa de Araticum apresenta alto valor energético, bem como elevados teores de fibras alimentares, provou ser uma fonte de vitamina $\mathrm{C}$, e uma excelente fonte de vitamina A e E. Conforme os resultados obtidos neste trabalho (Quadros 1 e 2), o processamento térmico para obtenção de doces de Araticum não prejudica o valor nutricional e energético do produto gerado.

O consumo de nutrientes de forma incorreta pode comprometer o estado nutricional e acarretar o desenvolvimento de carências ou excessos nutricionais, principalmente ao se tratar de crianças [21]. Nesse sentido, a vitamina A se destaca por sua função de proteção das estruturas e funções oculares, sistema imunológico e redução da morbimortalidade por doenças infecciosas, como diarreia, sarampo e malária. A hipovitaminose A, leva a problemas oculares como xeroftalmia e cegueira noturna [22].

Carvalho et al. (2015) [23] selecionaram 16 estudos, publicados entre 2003 e 2013, a respeito do consumo alimentar e adequação nutricional em crianças brasileiras. E notaram que a prevalência de inadequação de micronutrientes oscilou muito, para o ferro $(0,4 \%$ a $65 \%)$, para a vitamina A (20\% a 59,5\%), para o zinco (20\% a 99,4\%), para o cálcio (12,6\% a 48,9\%), e para a vitamina C $(9,6 \%$ a 96,6\%). Os doces de Araticum podem contribuir de forma positiva para o incremento da qualidade nutricional dos alimentos disponibilizados a crianças em idade escolar devido aos conteúdos de fibra alimentar, vitaminas A e C, carboidratos e proteínas (Quadros 1 e 2).

Quadro 1: Valores de informação nutricional das formulações 1 a 6 de doce em pasta de Araticum.

\begin{tabular}{|c|c|c|c|c|c|c|c|c|c|c|c|}
\hline \multicolumn{10}{|c|}{ INFORMAÇÃO NUTRICIONAL } \\
Porção de 20 g (1 colher de sopa)
\end{tabular}

Quanto à ingestão de nutrientes, é recomendado pelo Ministério da Saúde [13] e pelo Dietary reference intakes for energy, carbohydrate, fiber, fat, fatty acids, cholesterol, protein, and amino acids [24], um consumo diário de $25 \mathrm{~g}$ de fibra alimentar, $45 \mathrm{mg}$ de vitamina $\mathrm{C}$ e $600 \mu \mathrm{g}$ de vitamina A em dietas de $2000 \mathrm{kcal} \backslash d i a$ de valor energético. Porém em todas as regiões brasileiras o consumo destes elementos é deficiente, tanto por adultos quanto por crianças. As formulações de doces de Araticum desenvolvidas apresentaram 6,3 e $11 \mathrm{mg}$ de vitamina $C$ por porção de 20 gramas (Quadros 1 e 2), o que representa cerca de $25 \%$ do recomendo para ingestão diária.

O Araticum é uma alternativa alimentar que pode contribuir para o incremento de dietas especialmente em regiões com altos níveis de insegurança alimentar e em locais onde pode haver escassez ou ausência de alimentos considerados como fontes nutrientes na dieta brasileira [20]. Assim, o processamento de conservação em forma de doces implicaria em sua maior disponibilidade, possibilitando a inserção deste fruto na alimentação de crianças e jovens. 
Quadro 2: Valores de informação nutricional das formulações 7 a 11 de doce em pasta de Araticum.

\begin{tabular}{|c|c|c|c|c|c|c|c|c|c|c|}
\hline \multicolumn{11}{|c|}{$\begin{array}{l}\text { INFORMAÇÃ̃O NUTRICIONAL } \\
\text { Porção de } 20 \text { g ( } 1 \text { colher de sopa })\end{array}$} \\
\hline & \multicolumn{2}{|c|}{ Formulação 7} & \multicolumn{2}{|c|}{ Formulação 8} & \multicolumn{2}{|c|}{ Formulação 9} & \multicolumn{2}{|c|}{ Formulação 10} & \multicolumn{2}{|c|}{ Formulação 11} \\
\hline & $\begin{array}{c}\text { Qt. } \\
\text { porcão }\end{array}$ & $\begin{array}{c}\% \mathrm{VD} \\
(*)\end{array}$ & $\begin{array}{c}\text { Qt. } \\
\text { porcão }\end{array}$ & $\begin{array}{c}\% \mathrm{VD} \\
(*)\end{array}$ & $\begin{array}{l}\text { Qt. } \\
\text { porção }\end{array}$ & $\begin{array}{c}\% \mathrm{VD} \\
(*)\end{array}$ & $\begin{array}{l}\text { Qt. } \\
\text { porcão }\end{array}$ & $\begin{array}{c}\% \mathrm{VD} \\
(*)\end{array}$ & $\begin{array}{l}\text { Qt. } \\
\text { porcão }\end{array}$ & $\begin{array}{c}\% \mathrm{VD} \\
(*)\end{array}$ \\
\hline $\begin{array}{c}\text { Valor } \\
\text { Energético }\end{array}$ & $\begin{array}{c}80 \mathrm{Kcal}= \\
336 \mathrm{KJ}\end{array}$ & 4 & $\begin{array}{c}82 \mathrm{Kcal}= \\
343 \mathrm{KJ}\end{array}$ & 4 & $\begin{array}{c}60 \mathrm{Kcal}= \\
253 \mathrm{KJ}\end{array}$ & 3 & $\begin{array}{c}60 \mathrm{Kcal}= \\
253 \mathrm{KJ}\end{array}$ & 3 & $\begin{array}{c}60 \mathrm{Kcal}= \\
253 \mathrm{KJ}\end{array}$ & 3 \\
\hline Carboidratos & $19 \mathrm{~g}$ & 6 & $20 \mathrm{~g}$ & 7 & $14 \mathrm{~g}$ & 5 & $14 \mathrm{~g}$ & 5 & $14 \mathrm{~g}$ & 5 \\
\hline Proteínas & $0,1 \mathrm{~g}$ & 0 & $0,1 \mathrm{~g}$ & 0 & $0,2 \mathrm{~g}$ & 0 & $0,2 \mathrm{~g}$ & 0 & $0,2 \mathrm{~g}$ & 0 \\
\hline $\begin{array}{l}\text { Gorduras } \\
\text { Totais }\end{array}$ & $0,3 \mathrm{~g}$ & 1 & $0,3 \mathrm{~g}$ & 1 & $0,3 \mathrm{~g}$ & 1 & $0,3 \mathrm{~g}$ & 1 & $0,3 \mathrm{~g}$ & 1 \\
\hline $\begin{array}{c}\text { Fibra } \\
\text { Alimentar }\end{array}$ & $0,2 \mathrm{~g}$ & 1 & $0,3 \mathrm{~g}$ & 1 & $0,5 \mathrm{~g}$ & 2 & $0,5 \mathrm{~g}$ & 2 & $0,5 \mathrm{~g}$ & 2 \\
\hline Sódio & $0,5 \mathrm{mg}$ & 0 & $0,6 \mathrm{mg}$ & 0 & $0,7 \mathrm{mg}$ & 0 & $0,7 \mathrm{mg}$ & 0 & $0,7 \mathrm{mg}$ & 0 \\
\hline Cálcio & $2,3 \mathrm{mg}$ & 0 & $2,3 \mathrm{mg}$ & 0 & $3,3 \mathrm{mg}$ & 0 & $3,3 \mathrm{mg}$ & 0 & $3,3 \mathrm{mg}$ & 0 \\
\hline Vitamina A & $\begin{array}{l}5,8 \mathrm{mcg} \\
\mathrm{RE}\end{array}$ & 1 & $\begin{array}{l}5,9 \mathrm{mcg} \\
\mathrm{RE}\end{array}$ & 1 & $\begin{array}{l}6,3 \mathrm{mcg} \\
\mathrm{RE}\end{array}$ & 1 & $\begin{array}{l}6,3 \mathrm{mcg} \\
\mathrm{RE}\end{array}$ & 1 & $\begin{array}{l}6,3 \mathrm{mcg} \\
\mathrm{RE}\end{array}$ & 1 \\
\hline Vitamina C & $6,6 \mathrm{mg}$ & 15 & $6,7 \mathrm{mg}$ & 15 & $9,5 \mathrm{mg}$ & 21 & $9,5 \mathrm{mg}$ & 21 & $9,5 \mathrm{mg}$ & 21 \\
\hline
\end{tabular}

\section{CONCLUSÃO}

As formulações desenvolvidas apresentaram teor considerável de fibras alimentares e cálcio, além de serem fontes de vitamina $\mathrm{C}$ e A. Além disso, apresentaram baixos valores calóricos, baixo teor de gordura e sódio em comparação a produtos comercializados. Frente aos parâmetros nutricionais avaliados a melhor formulação foi a Formulação 1, mesmo apesentando valores de sódio e gordura pouco superior as demais. Os resultados apresentados neste trabalho reforçam que o desenvolvimento de doces com o fruto Araticum é uma alternativa viável de alimento para inserção na alimentação de crianças e jovens devido ao seu valor nutricional.

\section{REFERÊNCIAS BIBLIOGRÁFICAS}

1. Morzelle MC, Bachiega P, Souza EC, Boas V, De Barros EV, Lamounier ML. Caracterização química e física de frutos de curriola, gabiroba e murici provenientes do cerrado brasileiro. Rev Bras Fruticultura. 2015 Mar;(75):96-103. doi: 10.1590/0100-2945-036/14

2. Aguiar AO, Rodrigues DS, Sousa AR, Soares CMS, Ibiapina A, De Melo Filho AA, et al. Use of passion fruit albedo as a source of pectin to produce Araticum (Annona Crassiflora Mart.) preserves. Chem Engineering Transactions. 2019;(75):223-8. doi: 10.3303/CET1975038

3. Aguiar AO, De Souza ARM, Oliveira MOS. Araticum (Annona Crassiflora Mart.). In: Souza ARM, Daminani C, Martins GAS, Silva JFM, editores. Frutos do Cerrado: características e aplicações tecnológicas. Curitiba (PR): CRV; 2018. p. 85-96.

4. Freitas MLF, Menezes CC, Carneiro JDS, Reis RP. Diagnóstico do consumo e processo produtivo de doces de frutas produzidos artesanalmente. Alim Nitr. 2012;23(4):589-95.

5. Sousa AIO, Nóbrega JYL, Machado AV, Costa RO. Conservação de furtas através da utilização do açúcar. Rev Bras Agrotecnol. 2014;4(1):15-18.

6. Agência Nacional de Vigilância Sanitária; Universidade de Brasília. Rotulagem nutricional obrigatória: manual de orientação às indústrias de alimentos [Internet]. $2^{\mathrm{a}}$ versão. Brasília (DF): Ministério da Saúde, Agência Nacional de Vigilância Sanitária, Universidade de Brasília; 2005 [citado em 23 jul 2021]. Disponível em: https://www.gov.br/agricultura/pt-br/assuntos/inspecao/produtos-vegetal/legislacao1/biblioteca-de-normas-vinhos-e-bebidas/rotulagem-nutricional-obrigatoria-manual-de-orientacao-asindustrias-de-alimentos_2005.pdf/view.

7. Souza SMFC, Lima KC, Miranda HF, Cavalcanti FID. Utilização da informação nutricional de rótulos por consumidores de Natal, Brasil. Rev Panam Salud Publica. 2011;29(5):337-43.

8. Brasil. Lei n ${ }^{\circ} 8.078 / 90$, de 11 de setembro de 1990. Dispõe sobre a proteção do consumidor e dá outras providências. Diário Oficial da União. 12 set 1990;176(Seção 1; Suplemento):1-8. Disponível em: http://www.planalto.gov.br/ccivil_03/leis/18078compilado.htm

9. Câmara MCC, Marinho CLC, Guilam MC, Braga AMCB. A produção acadêmica sobre a rotulagem de alimentos no Brasil. Rev Panam Salud Publica. 2008;23(1):52-8. 
10. Silva IG, Martins GAS, Borges SV, Marques GR, Regis IS. Influence of passion fruit albedo, citric acid, and the pulp/sugar ratio on the quality of banana preserves. Food Sci Technol. 2012 Apr;32(2):267-73. doi:10.159/S0101-20612012005000038

11. Box GEP, Draper NR. Response surfaces, mixtures, and ridge analyses. 2nd ed. New York (US): John Wiley \& Sons; 2007.

12. Brasil. Mistério da Saúde, Agência Nacional de Vigilância Sanitária (Anvisa). Resolução-RDC n 359 , de 23 de dezembro de 2003. Anexo, Regulamento técnico de porções de alimentos embalados para fins de rotulagem nutricional. Diário Oficial da União. 26 dez 2003;251(Seção 1):28-32. Disponível em: https://bvsms.saude.gov.br/bvs/saudelegis/anvisa/2003/rdc0359_23_12_2003.html

13. Brasil. Mistério da Saúde, Agência Nacional de Vigilância Sanitária (Anvisa). Resolução-RDC n 360 , de 23 de dezembro de 2003. Anexo, Regulamento técnico sobre rotulagem nutricional de alimentos embalados. Diário Oficial da União. 26 dez 2003;251(Seção 1):33-4. Disponível em: https://bvsms.saude.gov.br/bvs/saudelegis/anvisa/2003/res0360_23_12_2003.html

14. Brasil. Mistério da Saúde, Secretaria de Vigilância Sanitária. Portaria ${ }^{\circ}$ 33, de 13 de janeiro de 1998: Anexo, Ingestão Diária Recomendada (IDR) para proteínas, vitaminas e minerais. Diário Oficial [da] República Federativa do Brasil. 16 jan 1998;11-E(Seção 1):5-6. Disponível em: https://bvsms.saude.gov.br/bvs/saudelegis/svs1/1998/prt0032_13_01_1998.html

15. Tabela Brasileira de Composição de Alimentos (TBCA), versão 6.0 [Internet]. São Paulo: Universidade de São Paulo (USP), Food Research Center (FoRC); 2017 [citado em 21 jul 2021]. Disponível em: http://www.tbca.net.br/base-dados/composicao_alimentos.php

16. US Departament of Agriculture (USDA), Agricultural Research Service, Nutrient Data Laboratory. Composition of foods raw, processed, prepared. USDA National Nutrient Database for Standard Reference, Legacy. Documentation and User Guide [Internet]. Beltsville (MD): USDA; 2018. Disponível em: https://www.ars.usda.gov/arsuserfiles/80400525/data/sr-legacy/sr-legacy_doc.pdf

17. Brasil. Ministério da Educação, Fundo Nacional de Desenvolvimento da Educação. Resolução nº 26, de 17 de junho de 2013. Dispõe sobre o atendimento da alimentação escolar aos alunos da educação básica no âmbito do Programa Nacional de Alimentação Escolar- PNAE. Diário Oficial da União. 18 jun 2013;115(Seção 1):7. Disponível em: https://www.in.gov.br/web/dou/-/resolucao-n-26-de-17-dejunho-de-2013-30683763

18. Arruda HS, Pereira GA, Pastore GM. Brazilian Cerrado fruit Araticum (Annona crassiflora Mart.) as a potential source of natural antioxidant compounds. Int Food Res J. 2018;25(5):2005-12.

19. Schiassi MCEV, De Souza VR, Lago AMT, Campos LG, Queiroz F. Fruits from the Brazilian Cerrado region: Physico-chemical characterization, bioactive compounds, antioxidant activities, and sensory evaluation. Food chem. 2018;245:305-11. doi: 10.1016/j.foodchem.2017.10.104

20. Cardoso L, Oliveira D, Bedetti S, Martino H, Pinheiro-Sant'ana H. Araticum (Annona crassiflora Mart.) from the Brazilian Cerrado: Chemical composition and bioactive compounds. Fruits. 2013;68(2):12134. doi: 10.1051/fruits/2013058

21. Cavalcante AAM, Tinôco ALA, Cotta RMM, Ribeiro RCL, Pereira CAS, Franceschini SCC. Consumo alimentar e estado nutricional de crianças atendidas em serviços públicos de saúde do município de Viçosa, Minas Gerais. Rev Nutrição. 2006;19(3):321-30. doi: 10.1590/S1415-52732006000300003

22. Paula WKAS, Caminha MFC, Figueirôa JN, Batista Filho M. Anemia e deficiência de vitamina A em crianças menores de cinco anos assistidas pela Estratégia Saúde da Família no Estado de Pernambuco, Brasil. Ciênc Saúde Coletiva. 2014;19(4):1209-22. doi: 10.1590/1413-81232014194.00602013

23. Carvalho CA, Fonsêca PCA, Priore SE, Franceschini SCC, Novaes JF. Consumo alimentar e adequação nutricional em crianças brasileiras: Revisão sistemática. Rev Paul Pediatr. 2015;33(2):211-21.

24. Institute of Medicine. Dietary Reference intakes for energy, carbohydrate, fiber, fat, fatty acids, cholesterol, protein, and amino acids. Washington (DC): The National Academies Press; 2005. doi: $10.17226 / 10490$ 